\title{
Desenvolvimento de um jogo para ensino de medição de software
}

\author{
Christiane Gresse von Wangenheim ${ }^{1,2}$, Marcello Thiry ${ }^{1}$, Djone Kochanski ${ }^{1}$, \\ Leonardo Steil ${ }^{1}$, Djoni Silva ${ }^{1}$, Juliana Lino ${ }^{3}$ \\ ${ }^{1}$ Ciência da Computação - CTTMAR \\ Universidade do Vale do Itajaí (UNIVALI) \\ São José - SC - Brasil \\ ${ }^{2}$ Departamento de Informática e Estatística \\ Universidade Federal de Santa Catarina (UFSC) \\ Florianópolis - SC - Brasil \\ ${ }^{3}$ Whirlpool S.A. \\ Joinville - SC - Brasil
}

\begin{abstract}
This paper describes the development and evaluation of an initial prototype of an educational game for software measurement. The game $X$ MED v1.0 focuses on the education of software measurement, simulating the definition and execution of a measurement program for project management in alignment with CMMI-DEV maturity level 2. In this paper, we present the conception of the game, its implementation and its evaluation through a series of experiments.
\end{abstract}

Resumo. Este artigo apresenta o desenvolvimento e avaliação de um protótipo inicial de um jogo educacional para medição de software. $O$ jogo $X$ MED v1.0 é voltado para o ensino de medição de software, simulando a definição e execução de um programa de medição voltado a gerência de projetos alinhado ao nível de maturidade 2 do CMMI-DEV. Neste artigo, são apresentadas a concepção do jogo, sua implementação e sua avaliação por uma série de experimentos.

\section{Introdução}

O reconhecimento dos potenciais benefícios da medição de software tem aumentado de forma significativa, mas o movimento da indústria de software ainda tem se mostrado tímido com relação ao estabelecimento de programas de medição. Além disso, o relato de insucessos em iniciativas de medição é freqüente (Dekkers e McQuaid, 2002; Kasunic, 2006). Resultados de uma pesquisa do SEI (Software Engineering Institute) também indicam que ainda há uma grande lacuna entre o existente e o desejado em termos de utilização de práticas de medição (SEI, 2006). Uma das razões apontadas é a falta de capacitação de profissionais (Hock e Hui, 2004), motivado pelo fato da medição ainda não ser um assunto contemplado de forma significativa na estrutura curricular de cursos da área de Computação e Informática. O resultado é que alunos de graduação e pós-graduação acabam conhecendo somente conceitos básicos em medição de software (Hock e Hui, 2004; Ott, 2005). Neste contexto, muitos cursos não conseguem 
demonstrar a relevância que a utilização da medição de software apresenta na prática, reduzindo o interesse dos educandos e deixando a impressão que o tema é difícil e complexo (Hock e Hui, 2004).

Uma das razões deste problema é a forma pela qual a medição de software é ensinada. Aulas expositivas, técnica predominante em todas as áreas da educação (Percival et. al., 1993), são adequadas para a apresentação de conceitos abstratos e relatos de fatos. Entretanto, elas não contribuem fortemente para atingir níveis cognitivos mais altos com ênfase na transferência de experiências para situações do mundo real (Choi e Hannafin, 1995). Além disso, como a medição representa somente uma área da Engenharia de Software, as disciplinas geralmente não têm muito espaço para oferecer tanto conhecimentos teóricos quanto práticos.

Por este motivo permanece o desafio de ensinar medição de uma forma concentrada e atraente, proporcionando o entendimento dos conceitos-chave e capacitando o educando para a aplicação de medição na prática.

Jogos educacionais têm se tornado uma alternativa para esta situação, oferecendo diversas vantagens (Percival et al., 1993). Jogos educacionais permitem a obtenção de uma equivalência virtual de experiências do mundo real. Desta forma, eles podem ser eficazes na complementação do ensino de conceitos básicos pela demonstração da aplicação de pontos relevantes, bem como no desenvolvimento de competências cognitivas pela aplicação de estudos de caso ilustrativos (Percival et al., 1993). Particularmente, jogos baseados em computador podem permitir o "aprender fazendo" em situações realistas, com fornecimento de feedback imediato. Isto pode tornar os educandos mais confiantes em suas habilidades para lidar com situações similares no mundo real. Além disso, a natureza envolvente dos jogos pode tornar o aprendizado mais divertido (Kafai, 2001).

Como a idéia de adotar jogos educacionais na Engenharia de Software é recente, não foram encontrados jogos para o ensino específico de medição de software durante a execução deste trabalho. Por outro lado, há diversos jogos em outras áreas da Engenharia de Software, principalmente, gerência de projetos. Exemplos são The Incredible Manager (Dantas et al., 2004), Project-o-poly (Buglione, 2007), Project Management Training Courses (Collofello, 2000) ou jogos de simulação para execução de processos de software, como, SimSE (Oh Navaro e van der Hoek, 2007) SESAM (Drappa e Ludewig, 2000), SimVBSE (Jain e Boehm, 2006), OSS (Sharp e Hall, 2000), Problems and Programmers (Baker et. al., 2003), SimulES (Figueiredo et. al., 2007) entre outros.

Neste contexto, foi iniciado o desenvolvimento de um jogo educacional para medição de software com o objetivo de reforçar a lembrança e a compreensão de conceitos básicos e treinar a aplicação de medição na prática. O jogo foi desenvolvido utilizando prototipação evolutiva baseada no modelo ADDIE - Analysis, Design, Development, Implementation and Evaluation (Molenda et. al., 1996). Seguindo este modelo foi iniciada a concepção e modelagem de uma versão inicial do jogo considerando uma análise de fatores críticos na aplicação de medição e em experiências práticas nesta área. Com base nesta concepção, desenvolveu-se um protótipo do jogo que foi avaliado por meio de uma série de experimentos como parte de um módulo de ensino sobre medição em três disciplinas de mestrado na área de Computação. 


\section{Concepção do Jogo Educacional X-MED v1.0}

O objetivo desta pesquisa é desenvolver um jogo educacional de simulação baseado em computador que simule a definição e execução de um programa de medição de software. O objetivo principal do jogo é exercitar a aplicação de medição de software voltada para a gerência de projetos alinhada ao nível 2 de maturidade do CMMI-DEV v1.2 (CMMI, 2006) ou o respectivo nível F do modelo MSP.BR (SOFTEX, 2007). O processo de medição adotado no jogo é baseado no GQM - Goal/Question/Metric (Basili et al., 1994) e inclui elementos do PSM - Practical Software and Systems Measurement (McGarry et al., 2001).

O público alvo do jogo é formado por alunos de pós-graduação em cursos de Computação e/ou profissionais de Engenharia de Software. O jogo é projetado como um complemento às aulas tradicionais ou cursos de e-learning provendo um ambiente para exercitar os conceitos apresentados. É suposto que os alunos tenham conhecimento básico em Engenharia de Software, medição de software, gerência de projetos e sobre CMMI ou MPS.BR.

O Quadro 1 apresenta um plano de ensino exemplo, que ilustra o contexto educacional previsto para a aplicação do jogo.

\section{Quadro 1. Exemplo de plano de ensino para a aplicação do jogo X-MED v1.0}

\section{Plano de ensino do módulo “Medição de software” \\ Contexto. Este módulo foi planejado com parte de um curso de pós-graduação ou de um treinamento profissional relacionado à Engenharia de Software, Qualidade de Software ou Melhoria de Processo de Software.}

Pré-requisitos. Espera-se que os alunos sejam graduados em Ciência da Computação (ou em área afim) e que tenham uma compreensão básica sobre Engenharia de Software, Gerência de Projetos e ainda sobre o modelo CMMI e/ou MPS.BR.

Objetivo do módulo. Prover uma compreensão básica sobre a definição e execução de programas de medição de software para gerência de projetos alinhados ao nível 2 de maturidade do CMMI-DEV e/ou nível F do MSP.BR.

Ementa. Conceitos e terminologia de medição. Visão geral da norma ISO/IEC 15939. Visão geral sobre métodos de medição (GQM, PSM). Modelos de referência de medição (CMMI, MPS.BR). Processo de medição passo a passo: caracterização de contexto, definição de objetivos de medição, desenvolvimento do plano GQM, desenvolvimento do plano de coleta, verificação e armazenamento de dados, análise e interpretação de dados, comunicação dos dados e dos resultados de medição.

Resultados de aprendizagem. Como resultado deste módulo, o aluno deve ter uma compreensão básica sobre medição de software e deverá ser capaz de definir e executar programas básicos de medição (sob supervisão) para gerência de projetos. O aluno deverá ainda ter uma compreensão básica das práticas/resultados requeridos pelos modelos de referência no processo de medição. O objetivo de aprendizagem deste módulo é voltado para aprendizagem cognitiva, incluindo conhecimento declarativo e procedural, considerando os níveis de lembrança, compreensão e aplicação de acordo com a versão revisada da taxonomia de Bloom (Anderson and Krathwohl, 2001). Além disso, o módulo também é construído para permitir a mudança de atitude do aluno, aumentando seu reconhecimento sobre a importância da medição na prática, caracterizando uma maior motivação e interesse pela medição.

\begin{tabular}{|l|l|}
\hline CONTEÚDO & ESTRATÉGIA DE ENSINO \\
\hline Unidade 1 - Conceitos básicos & Aula expositiva com discussão \\
\hline $\begin{array}{l}\text { Unidade } 2 \text { - Processo de medição passo a } \\
\text { passo }\end{array}$ & $\begin{array}{l}\text { Aula expositiva com discussão, exercícios em sala } \\
\text { de aula, jogo X-MED v1.0 }\end{array}$ \\
\hline
\end{tabular}


Avaliação. O grau de atendimento dos resultados esperados de aprendizagem é avaliado por prova(s) escrita(s) com perguntas de múltipla escolha. As provas incluem perguntas em todos os três níveis de conhecimento (lembrança, compreensão e aplicação).

Neste contexto, foram identificados os seguintes requisitos de alto nível referente à concepção do jogo:

1. O jogo deve simular a aplicação prática de um programa de medição com foco em gerência de projetos alinhado ao nível de maturidade 2 do modelo CMMIDEV ou ao respectivo nível F do MPS.BR.

2. O escopo do jogo deve incluir todos os passos de um programa de medição, incluindo a definição e execução com base na abordagem GQM e com a utilização de elementos do PSM.

3. O aluno deve poder jogar em modo individual (single-player), sem necessidade de interação com outros alunos e/ou um instrutor.

4. A duração média de uma sessão do jogo deve ser em torno de 2 horas.

5. O jogo deve fornecer feedback ao aluno sobre o seu desempenho com base em critérios de avaliação pré-definidos.

6. O jogo deve ser disponibilizado para livre utilização.

7. O jogo deve ser em língua portuguesa.

O objetivo específico de aprendizagem do jogo é reforçar os conceitos de medição e prover competência na aplicação dos conhecimentos obtidos, atingindo os níveis cognitivos de lembrança, compreensão e aplicação de acordo com a versão revisada da taxonomia de objetivos educacionais de Bloom (Anderson e Krathwohl, 2001). A Figura 1 apresenta uma versão revisada da taxonomia adotada, identificando os níveis cognitivos de interesse.

6. Criação: reunir elementos para formar um todo coerente ou funcional; reorganizar elementos em um novo padrão ou estrutura.

5. Avaliação: julgar com base em critérios e padrões.

4. Análise: decompor um elemento em suas partes constituintes e determinar seus princípios de organização.

3. Aplicação: utilizar um procedimento aprendido em novas situações.

2. Compreensão: construir significado a partir de mensagens educacionais, incluindo a comunicação oral, escrita e gráfica.

1. Lembrança: recuperar conhecimentos relevantes, como fatos, conceitos e padrões, da memória de longo prazo.

Figura 1: Versão revisada da taxonomia de Bloom. Fonte: Anderson e Krathwohl (2001).

O primeiro passo dado nessa direção foi o desenvolvimento do X-MED v1.0 (LINO, 2007). O X-MED v1.0 representa uma versão simplificada de um jogo educacional de 
simulação. Por meio da seleção de soluções adequadas às tarefas de medição, entendese que os alunos possam aprender como desenvolver ou selecionar objetivos de medição, planos GQM, planos de coleta de dados e a verificação, análise e interpretação de dados.

Esta versão do jogo segue um fluxo narrativo linear em que o aluno assume o papel de um analista de medição e segue seqüencialmente todas as tarefas de um programa de medição. A estrutura básica do jogo é apresentada no Quadro 2.

\section{Quadro 2. Estrutura básica do X-MED v1.0}

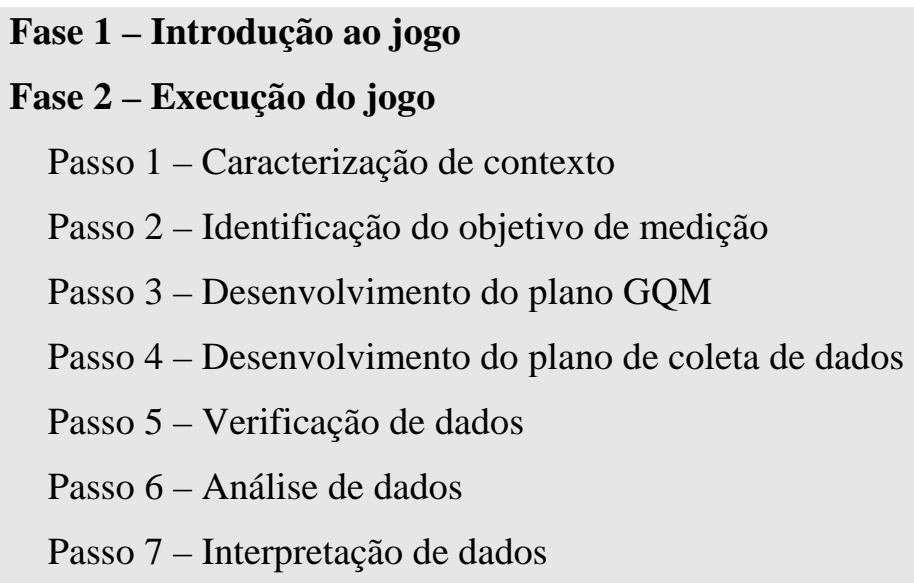

Fase 3 - Finalização do jogo

Em cada passo, uma tarefa é apresentada ao aluno. Por exemplo, no passo 2, o jogo pede ao aluno para que ele identifique o objetivo de medição mais apropriado para a situação dada (Figura 2). Para apoiar a decisão do aluno, o jogo apresenta instruções, materiais (p.ex., descrição do produto, registros de entrevistas), etc. Seguindo o exemplo do passo 2, o jogo mostra um relato de uma reunião de brainstorming para a identificação de objetivos de medição na empresa hipotética. Após a análise do material apresentado, o aluno deve selecionar uma solução para esta tarefa. Para cada tarefa, o jogo apresenta seis possíveis soluções como alternativas e o aluno deve escolher a mais adequada. Por exemplo, no passo 2, a tarefa é selecionar o objetivo de medição mais adequado na situação dada entre os seis objetivos apresentados pelo jogo. Imediatamente após o aluno selecionar uma das alternativas, o jogo fornece um feedback e uma pontuação pré-definida. Independente de qual alternativa for selecionada pelo aluno, o jogo segue para o passo seguinte, utilizando sempre a alternativa correta do passo anterior como entrada. No final, uma pontuação total é calculada somando as pontuações parciais e é gerado um relatório final. 


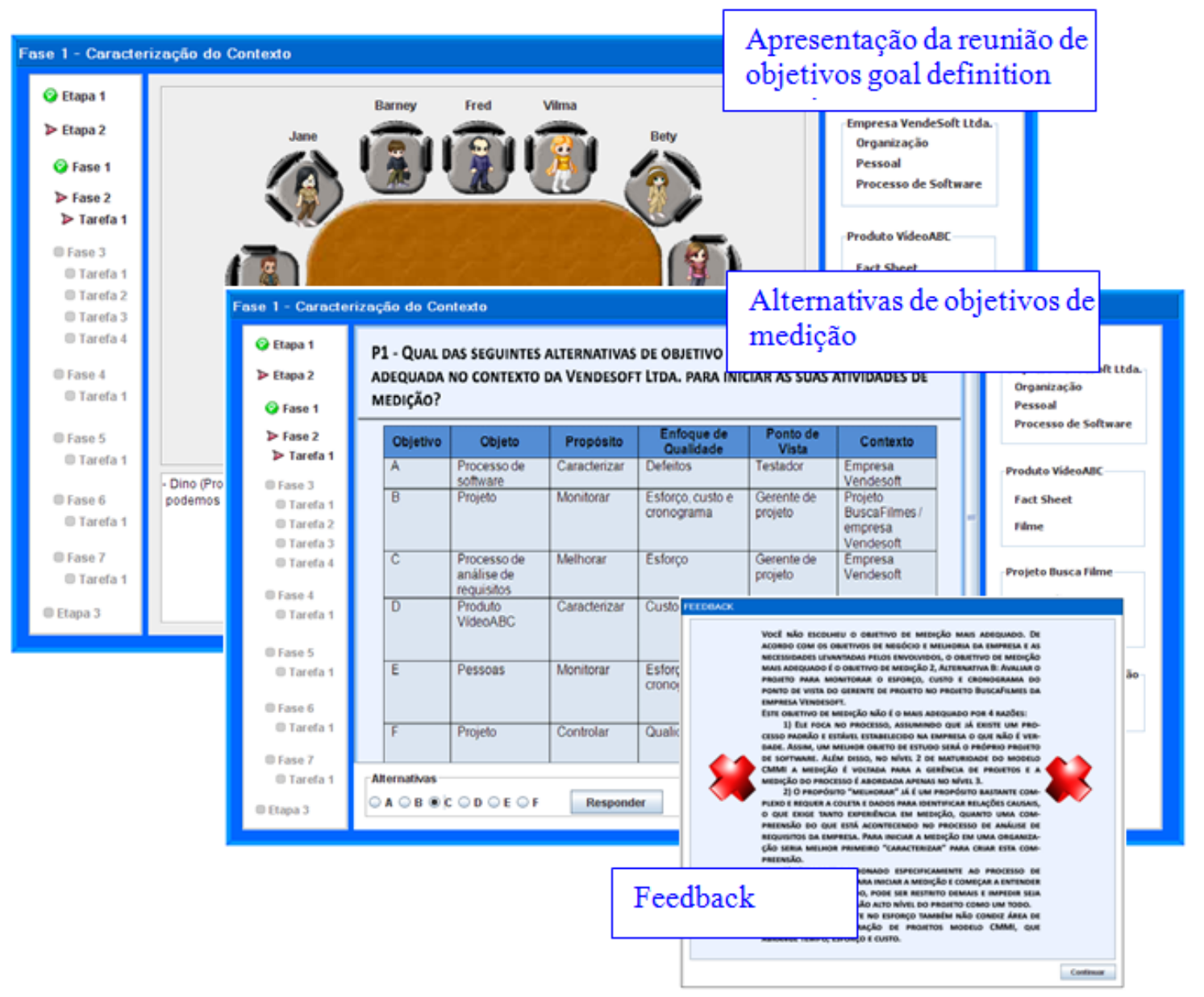

Figura 2: Exemplos de telas do X-MED v1.0

Todo o material do jogo foi preparado com base na literatura sobre medição, experiências reportadas e sobre as experiências dos autores na aplicação de medição na prática. Neste momento, o X-MED v1.0 possui apenas um cenário estático voltado para a aplicação de medição de software no gerenciamento de projetos. Não é possível configurar ou customizar outras abordagens de medição ou cenários.

Desta forma, o X-MED v1.0 representa uma versão simplificada e limitada de um jogo educacional de simulação. Embora existam diferentes perspectivas sobre jogos, o XMED v1.0 pode ser considerado um jogo de acordo com uma definição comum (Dempsey et. al., 1996). Embora simples, ele oferece uma competição/desafio (para a obtenção de uma pontuação máxima) na qual o aluno opera sobre regras de acordo com a teoria da medição para alcançar um objetivo de aprendizagem (definir e executar adequadamente, partes de um programa de medição). O X-MED v1.0 pode ser considerado também um "jogo utilizado como estudo de caso” (Ellington et al., 1982), uma vez que o aluno precisa examinar um cenário realista onde ele assume o papel de analista de medição em uma organização de software hipotética.

Nesta primeira versão do jogo, o fluxo de eventos é linear, sendo estruturado em seleções de alternativas pré-definidas e seguindo os passos de um programa de medição GQM. Entretanto, apoiado no trabalho de (Greitzer et al., 2007), este trabalho considera que mesmo uma experiência de aprendizagem relativamente simples pode ser uma 
oportunidade significativa para o aprendizado por meio do foco em um problema e de decisões relacionadas à conteúdo.

\section{Implementação do Protótipo XMED v1.0}

Do ponto de vista da execução do programa, o jogo é essencialmente seqüencial. A Figura 3 apresenta um diagrama de atividades, que representa o fluxo de controle do jogo. A animação indicada é bastante simples, mas oferece uma interface para o jogador visualizar situações como uma entrevista.

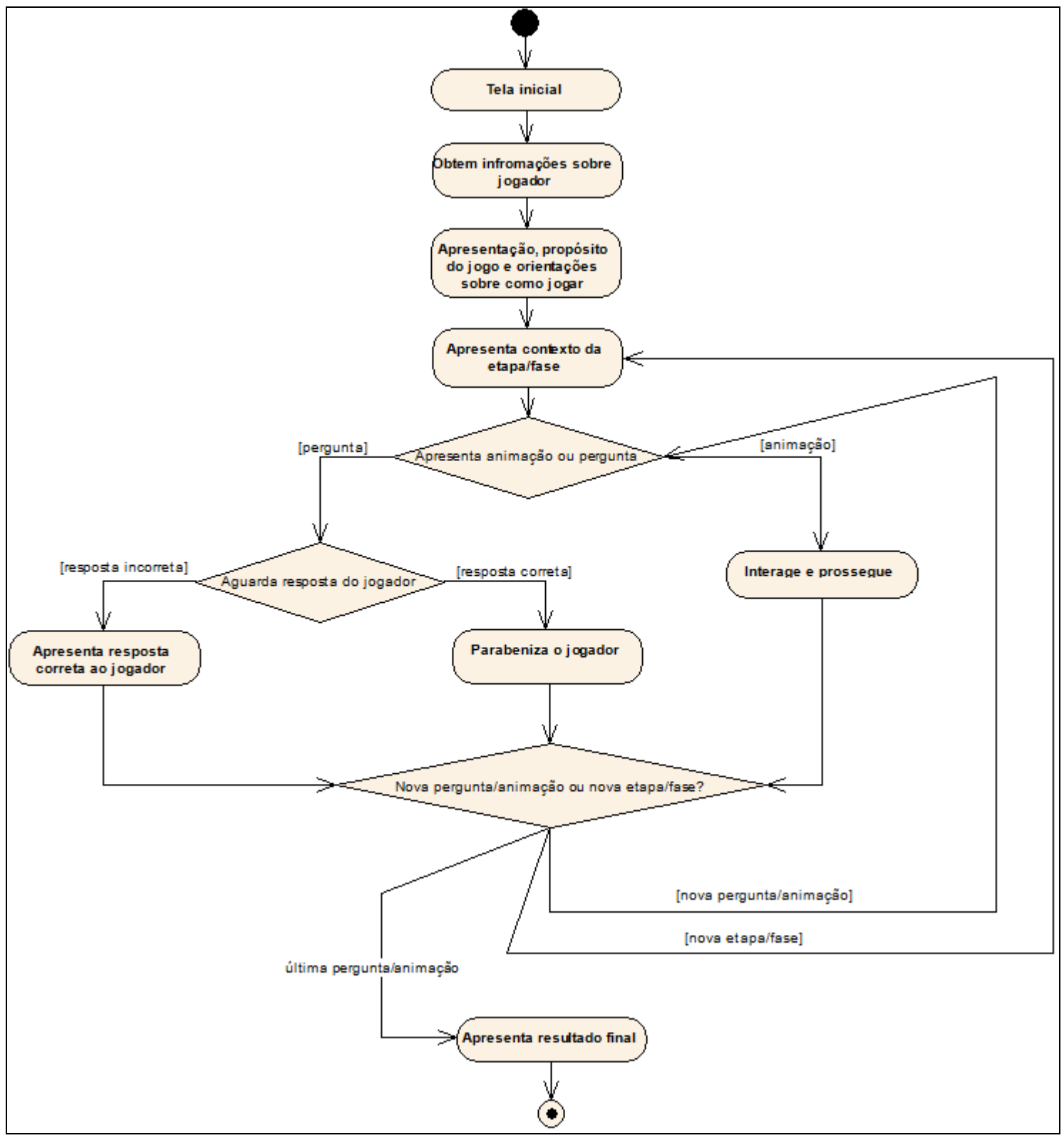

Figura 3: Fluxo de atividades no X-MED v1.0

Baseado na concepção do jogo, um protótipo para desktop foi implementado em Java utilizando o JDK 6. Esta versão ainda não integra elementos de multimídia, como, p.ex. áudio, animações, etc. 
O jogo foi desenvolvido a partir de uma arquitetura em camadas (Jacobson et al, 1992), utilizando classes de limite (interface com o usuário), classes de controle (regras de negócio e fluxos de controle do programa) e classes de entidade (armazenamento dos dados). Estes conceitos permitem a distribuição de responsabilidades em um conjunto de elementos de projeto interagindo entre si baseado em três perspectivas chave de uma colaboração (Figura 4).

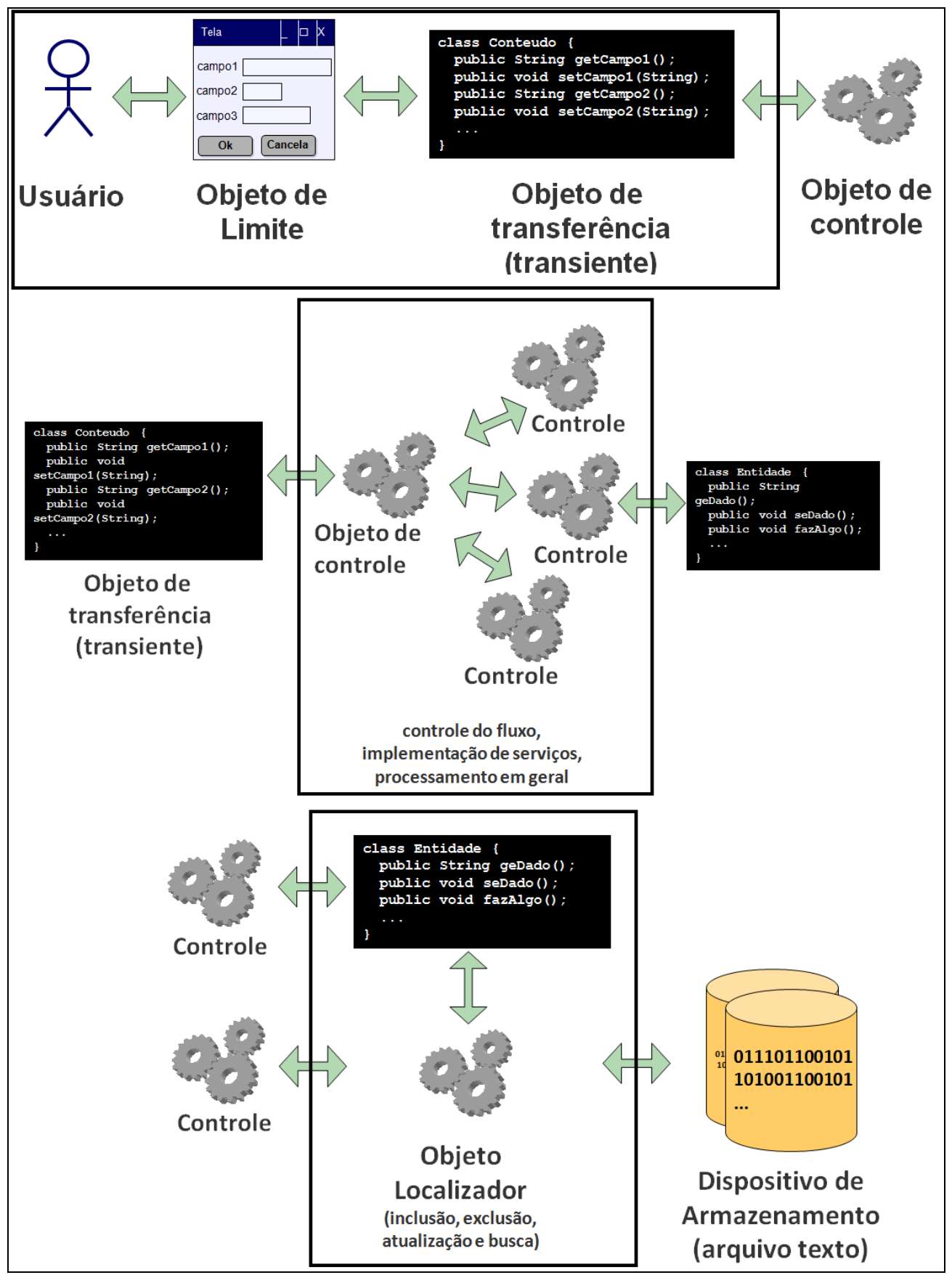

Figura 4: Arquitetura em camadas adotada no X-MED v1.0

A instalação do jogo é simples, uma vez que não ela não exige a configuração de banco de dados ou algum software externo. Como a estrutura de armazenamento é desenvolvida internamente, a instalação é a cópia direta dos arquivos para a máquina do 
jogador. O X-MED v1.0 está disponível para download em HTTP://www.incremental.com.br/xmed.

\section{Avaliação}

Esta seção apresenta, de forma resumida, a definição e resultados da avaliação do jogo $\mathrm{X}$-MED v1.0. Mais detalhes, incluindo todo material utilizado e todos os dados coletados estão detalhados em (Gresse von Wangenheim et al., 2008).

Para obter um primeiro feedback sobre a efetividade do protótipo do jogo X-MED como ferramenta educacional, foi realizada uma série de experimentos. O principal objetivo de pesquisa analisado foi se a utilização do jogo educacional X-MED v1.0 teve um efeito de aprendizagem positivo na capacidade dos alunos em definir e executar programas de medição para a gerência de projetos alinhada ao nível 2 de maturidade do CMMI-DEV e/ou nível F do MPS.BR. Esperava-se, que houvesse um efeito positivo de reforço referente ao nível de lembrança e compreensão de conceitos de medição e um efeito de aprendizagem positivo na capacidade de aplicação dos conhecimentos adquiridos. O segundo objetivo deste estudo foi avaliar se o jogo é apropriado, em termos de conteúdo (relevância, corretude, suficiência, dificuldade e seqüência), método de ensino e duração, bem como sua atratividade do ponto de vista dos alunos. Também se pretendeu obter um feedback relativo aos pontos fracos e fortes do protótipo do jogo.

Foi planejada e executada uma série de experimentos no contexto de um módulo de ensino sobre medição de software em disciplinas de mestrado. Com o objetivo de obter um número maior de participantes, o mesmo experimento foi executado em paralelo, como parte das seguintes disciplinas:

- Melhoria de Processos de Software durante o terceiro trimestre de 2007 do Programa de Pós-Graduação em Ciência da Computação da UFSC.

- Engenharia de Software durante o segundo semestre de 2007 do curso de Mestrado em Computação Aplicada da UNIVALI.

- Qualidade e Produtividade de Software durante o segundo semestre de 2007 do curso de Mestrado em Computação Aplicada da UNIVALI.

Ao todo, 15 alunos participaram e completaram a série de experimentos. Em cada experimento foi aplicado um design de experimento clássico (ver Quadro 3), ou seja, design de grupo de controle com pré-teste e pós-teste e seleção aleatória (Wohlin et al., 2000).

\section{Quadro 3 - Design de experimento adotado para avaliação do X-MED v.1.0}

\begin{tabular}{lccccc}
\multicolumn{1}{c}{ Grupo } & Seleção & Treinamento & Pré-teste & Tratamento & Pós-teste \\
(A) Experimental & Aleatória & Aula e exercícios & Prova 1 & Jogo & Prova 2 \\
(B) Controle & Aleatória & Aula e exercícios & Prova 1 & - & Prova 2
\end{tabular}

Objetivando minimizar o problema de desvios, a distribuição dos participantes para o grupo experimental e de controle foi aleatorizado de maneira balanceada. Em cada experimento, os grupos foram igualmente treinados em conceitos básicos de medição e no processo de medição por meio de aulas expositivas e exercícios em sala de aula pelo mesmo educador. Após estas aulas, os dois grupos, em cada experimento, realizaram o pré-teste. Em seguida, apenas o grupo experimental aplicou o jogo XMED v1.0. O 
grupo de controle não recebeu nenhum tratamento. No final, os dois grupos, novamente para cada experimento, realizaram o pós-teste.

O cronograma e os meios de coleta de dados sobre a execução da avaliação são apresentados no Quadro 4.

Quadro 4 - Cronograma e meios de coleta dos experimentos

\begin{tabular}{|c|c|c|c|}
\hline Dia & Conteúdo & Meios de coleta & Duração \\
\hline \multirow{4}{*}{1} & Apresentação do plano de ensino & $5 \mathrm{~min}$ \\
\cline { 2 - 4 } & & Formulário de consentimento & $5 \mathrm{~min}$ \\
\cline { 2 - 4 } & & $\begin{array}{c}\text { Questionário sobre os } \\
\text { participantes (formação) }\end{array}$ & $10 \mathrm{~min}$ \\
\cline { 2 - 4 } & Aula e exercícios & & $3: 30 \mathrm{~h}$ \\
\cline { 2 - 4 } & & Questionário pós-aula & $15 \mathrm{~min}$ \\
\hline \multirow{2}{*}{2} & Pré-teste & Questionário de teste & $1 \mathrm{~h}$ \\
\cline { 2 - 4 } & & Arquivo de log do jogo & $2 \mathrm{hin}$ \\
\hline \multirow{2}{*}{3} & Jogo (somente grupo do experimento) & Questionário pós-jogo & $30 \mathrm{~min}$ \\
\cline { 2 - 4 } & & & $1 \mathrm{~h}$ \\
\hline \multirow{2}{*}{4} & Pós-teste & Questionário do teste & $5 \mathrm{~min}$ \\
\cline { 2 - 4 } & & & \\
\hline
\end{tabular}

Para esta avaliação do jogo foram elaboradas as seguintes perguntas de pesquisa:

- Pergunta de pesquisa 1: o efeito de aprendizagem nos níveis de lembrança, compreensão e aplicação no grupo experimental A são superiores aos do grupo de controle B?

- Pergunta de pesquisa 2: o jogo educacional é considerado apropriado em termos de relevância de conteúdo, corretude e grau de dificuldade, seqüência, método de ensino e duração no contexto para o qual é destinado? O jogo é considerado atrativo? Quais são seus pontos fortes e fracos?

O objetivo destas perguntas de pesquisa foi obter uma avaliação subjetiva destes aspectos do ponto de vista dos alunos, ao invés de uma avaliação formal. Com respeito a uma análise compreensiva da corretude, relevância e completude do jogo, revisões foram realizadas por especialistas em engenharia de software durante 0 desenvolvimento do jogo.

\subsection{Análise e discussão}

Como resultado do experimento, muitos participantes acreditaram de modo subjetivo que o jogo os ajudou a aprender tanto sobre conceitos e processo quanto sobre a aplicação da medição (Figura 5). 


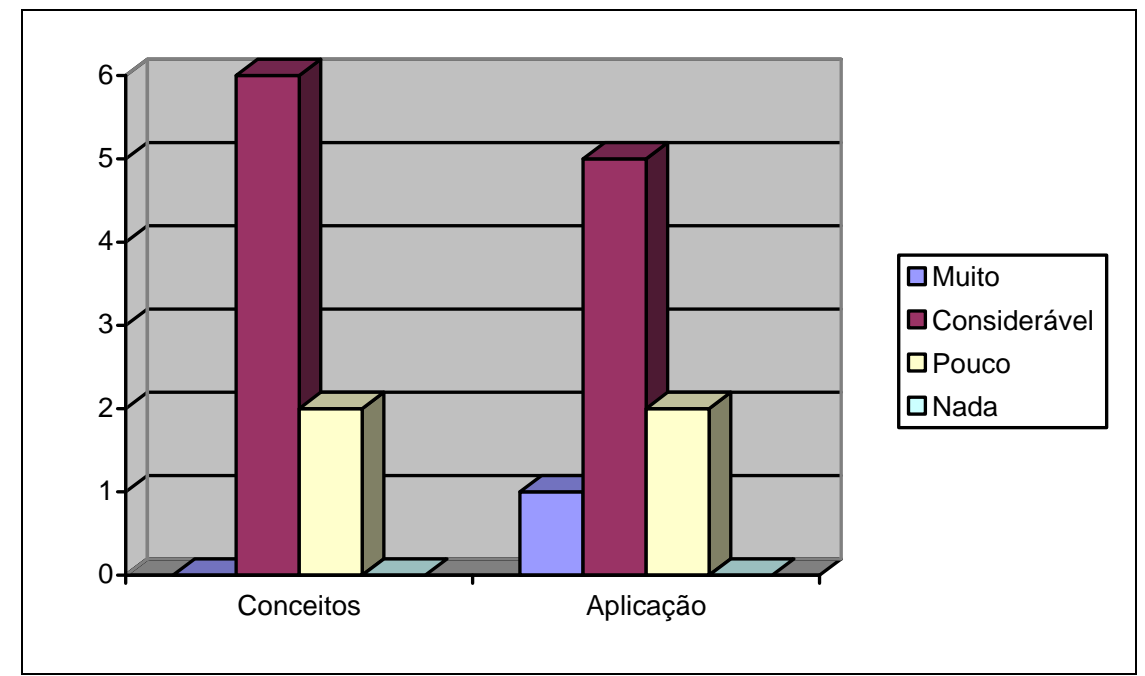

Figura 5: Resultados do efeito subjetivo de aprendizagem $(n=8$, somente grupo do experimento)

Porém, os resultados dos testes estatísticos não apoiaram esta avaliação subjetiva. Nenhuma diferença significativa na efetividade do aprendizado entre os grupos do experimento e de controle puderam ser identificadas. A análise utilizou o teste unilateral U Mann-Whitney com nível de significância $\alpha=0,05$. A explicação mais direta para estes resultados é que o jogo nesta versão 1.0 não é adequado para suportar efetivamente a aprendizagem.

Outra explicação é que o grau de dificuldade do jogo requer um entendimento maior sobre medição de software, gerência de projetos e do CMMI/ MPS.BR do que os alunos puderam adquirir durante uma aula com 4 horas de duração. Vários participantes comentaram que a experiência da aula foi bastante densa e sugeriram aumentar a duração da aula antes de jogar o jogo. No experimento, os participantes jogaram o jogo em sala de aula sem intervalo. A média de tempo para jogar o jogo foi aproximadamente 100 minutos, bem acima do tempo típico de concentração de 20 minutos. Então, em função da duração os participantes podem ter perdido a concentração e motivação. Isto pode ter sido provocado também pelo formato atual do protótipo, utilizando apenas texto, bem como a quantidade de alternativas para respostas às perguntas.

Outras causas podem estar relacionadas às características do experimento. Uma razão é que devido à pequena amostragem não foi possível identificar resultados estatisticamente significantes. Além disso, o pequeno número de questões no pré e pósteste podem ter dificultado a observação da dimensão dos efeitos. Outro fator, que pode ter influenciado a pontuação dos testes é o tempo adicional que os participantes de ambos os grupos gastaram estudando para o teste. Baseado nas informações dos participantes, os participantes do grupo A estudaram um pouco mais para o teste do que os participantes do grupo B, o qual poderia causar um efeito de aprendizagem maior no grupo A.

De qualquer forma, comparando a pontuação dos testes com a avaliação subjetiva da competência, um aspecto interessante pode ser observado. Dois participantes do grupo experimental e um participante do grupo de controle avaliaram de forma subjetiva a sua competência em medição menor no pós-teste do que no pré-teste, embora sua pontuação 
nos testes tenha melhorado. Isto pode indicar, que o teste, bem como o jogo, contribuíram em uma auto-avaliação mais realista.

Em geral, os participantes avaliaram o protótipo do jogo como apropriado e atrativo. Embora o protótipo seja muito simples, o foco principal foi obter um primeiro feedback de forma rápida. Muitas sugestões de melhoria importantes foram relatadas para o uso de elementos multimídia como forma de aumentar a atratividade do jogo. E, embora muitos participantes não tenham achado divertida a experiência do jogo, eles comentaram que preferiram o jogo em relação a exercícios tradicionais. As melhorias em aspectos de atratividade e fatores de diversão do jogo podem ajudar também na melhoria dos impactos de aprendizagem. Um dos pontos mais fortes do jogo foi o fornecimento de um feedback detalhado imediatamente após cada decisão, que ajudou os alunos a entenderem melhor o assunto.

Resultados como os obtidos nesse estudo, podem ser observados também em outros trabalhos relacionados. Por exemplo, no estudo do sistema SESAM (Drappa e Ludewig, 2000) um efeito de aprendizagem também não pôde ser confirmado. Isto pode ser explicado pelo fato de que a avaliação no domínio da educação é difícil. Devido à interação de múltiplos fatores, é freqüentemente impossível isolar os efeitos de uma técnica educacional, para obter o real efeito de aprendizagem longitudinalmente e/ou obter resultados estatisticamente significantes (Oh Navarro e van der Hoek, 2007). Além disso, a imaturidade do domínio da Engenharia de Software cria dificuldades para a condução de avaliações comparativas (Oh Navarro e van der Hoek, 2007).

\section{Conclusão}

Neste artigo, foi apresentado o desenvolvimento e avaliação da versão inicial do jogo educacional X-MED v1.0 e embora a avaliação não tenha mostrado estatisticamente os efeitos de aprendizagem, as avaliações subjetivas indicaram o potencial do jogo para a educação. O estudo permitiu também obter um primeiro feedback sobre o jogo bem como seus pontos fortes e fracos, para direcionar sua evolução. Apesar dos resultados terem permitido responder apenas algumas das perguntas iniciais, eles também levantaram novas questões e apresentaram detalhes que precisam ser observados em novos experimentos. Para isso, está sendo planejada a repetição do experimento com certas modificações no treinamento inicial, nos materiais para o experimento e no jogo em si.

Com base nos feedbacks obtidos, uma nova versão do jogo está em desenvolvimento para permitir a evolução de sua complexidade para torná-lo um jogo de simulação mais avançado. Isto inclui, principalmente, modificações na seqüência não-linear das ações por meio da construção de um motor de simulação e, conseqüentemente, no aumento de opções e de cenários disponíveis. Espera-se que estas modificações possam permitir uma experiência de aprendizado mais dinâmica e possibilitar a execução de várias sessões do jogo sem repetição de cenários. Além disso, o design do jogo está sendo melhorado, com base em frameworks de usabilidade, incluindo elementos multimídia, como animações das entrevistas e reuniões simuladas no jogo. Com a inclusão destas modificações, a nova versão do jogo será para a Web. Uma vez que a versão melhorada do jogo esteja disponível, o experimento será repetido. Neste contexto, os resultados da pesquisa apresentada neste artigo serão também válidos como uma linha base para comparações. 


\section{Agradecimentos}

Os autores gostariam de agradecer aos alunos dos cursos de mestrado que participaram do experimento. Agradecimentos também à Emily Oh Navarro por compartilhar materiais e à Sílvia M. Nassar por suas orientações nas análises estatísticas.

Este trabalho é apoiado pelo CNPq (Conselho Nacional de Desenvolvimento Científico e Tecnológico) e pela UNIVALI - Universidade do Vale do Itajaí.

\section{Referências}

Anderson, L. W. e Krathwohl, D. R. (Eds.). (2001). “A Taxonomy for Learning, Teaching, and Assessing: A Revision of Bloom's Taxonomy of Educational Objectives”. New York: Longman.

Baker, A., et. al. (2003) "Problems and Programmers: An Educational Software Engineering Card Game”. Proc. of the 2003 International Conference on Software Engineering, Portland, Oregon. p. 614-619.

Basili, V. R., et. al. (1994) “Goal/Question/Metric Approach”. In J. Marciniak (ed.), Encyclopedia of Software Engineering, vol. 1. John Wiley \& Sons.

Buglione, L. (2007) "Project-o-poly. Giocare per Apprendere. Il gioco come opportunità nelle Learning Organizations”. Persone \& Conoscenze, Jan/Feb 2007, No.26/27, ESTE, pp 43-47 (em Italiano).

Choi J, Hannafin M (1995) "Situated Cognition and Learning Environments: Roles, Structures and Implications for Design”. Educational Technology Research and Development, 43(2), pp 53-69.

CMMI Product Team (2006) “CMMI for Development, Version 1.2. Technical Report” CMU/SEI-2006-TR-008, Software Engineering Institute/ Carnegie Mellon University, Pittsburgh, Pennsylvania.

Collofello, J. S. (2000) "University/Industry Collaboration in Developing a Simulation Based Software Project Management Training Course”. Proc. of the 13th Conference on Software Engineering Education and Training, Austin, Texas, pp 161-168.

Dantas, A., Barros, M. e Werner, C (2004) “A Simulation-Based Game for Project Management Experiential Learning”. Proc. of the 16th International Conference on Software Engineering \& Knowledge Engineering (SEKE'2004), Banff, Canada, pp. $19-24$.

Dekkers, C. A. e McQuaid, P. A. (2002) "The Dangers of Using Software Metrics to (Mis)Manage”. IEEE IT Professional, IEEE Computer Society, vol. 4, no. 2, March /April 2002.

Dempsey, J. V., Lucassen, B. e Rasmussen, K. (1996) “The Instructional Gaming Literature: Implications and 99 Sources”. College of Education, University of South Alabama. Technical Report 96-1.

Drappa, A. e Ludewig, J. (2000) “Simulation in Software Engineering Training”. Proc. of the 22nd International Conference on Software Engineering. Limerick, Ireland, pp 199-208. 
Ellington H, Addinall E, Percival, F (1982) A Handbook of Game Design., Kogan Page, London

Figueiredo, E., Lobato, C., Dias, K., Leite, J. e Lucena, C. (2007) Um Jogo para o Ensino de Engenharia de Software Centrado na Perspectiva de Evolução. Anais do XV Workshop sobre Educação em Computação (WEI) co-alocado ao XXVII Congresso da SBC. Rio de Janeiro, pp. 37-46

Greitzer, F. L., Kuchar, O. A., Huston, K. (2007) Implications for Enhancing Training Effectiveness in a Serious Gaming Context. ACM Journal of Educational Resources in Computing, Vol. 7, No. 3, Art. 2.

Gresse von Wangenheim, C., Thiry, M., Kochinski, D. (2008). Empirical evaluation of an educational game on software measurement. Empirical Software Engineering, Springer Netherlands.

Hock, G. T. e Hui, G. L. S. (2004) “A Study of the problems and challenges of applying software metrics in software development industry”. Proc. of the M2USIC - MMU International Symposium on Information and Communication Technologies, Putrajaya, Malaysia.

Jacobson, I, Christerson, M, Jonsson, P. e Overgaard, G. (1992) “Object-Oriented Software Engineering: A Use Case Driven Approach”. ACM Press, AddisonWesley.

Jain, A. e Boehm, B. (2006) "SimVBSE: Developing a Game for Value-Based Software Engineering”. Proc. of the 19th Conference on Software Engineering Education and Training. Turtle Bay, Hawaii, pp 103-111.

Kafai, Y. B. (2001) “The Educational Potential of Electronic Games: From Games-ToTeach to Games-To-Learn”. Conference on Playing by the Rules: The Cultural Policy Challenges of Video Games, Chicago, Illinois.

Kasunic, M. (2006) “The State of Software Measurement Practice: Results of 2006 Survey”. Technical Report CMU/SEI-2006-TR-009, Carnegie Mellon University/ Software Engineering Institute, Pittsburgh, Pennsylvania.

Lino, J. I. (2007) "Proposta de um Jogo Educacional para a área de Medição e Análise de Software”. Trabalho de Conclusão de Curso (Graduação em Sistemas de Informação) - Universidade Federal de Santa Catarina, Florianópolis.

McGarry, J. et. al.. (2001) "Practical Software Measurement: Objective Information for Decision Makers”. Addison-Wesley Professional.

Molenda, M., Pershing, J. A. e Reigeluth, C. M. (1996) "Designing Instructional Systems”. In R. L. Craig (Ed.), The ASTD training and development handbook 4th ed. McGraw-Hill, New York, pp. 266-293.

Oh Navarro, E. e van der Hoek, A. (2007) "Comprehensive Evaluation of an Educational Software Engineering Simulation Environment”. Proc. of the 20th Conference on Software Engineering Education and Training, Dublin, Ireland, pp 195-202. 
Ott, L. M. (2005) "Developing Healthy Skepticism not Disbelief - Problems in Teaching Software Metrics". Proceedings of the 1st Workshop on Methods for Learning Metrics at the 11th IEEE Software Metrics Symposium. Como, Italy.

Percival, F., Ellington, H. e Race, P. (1993) “Handbook of Educational Technology”. 3. Ed., Kogan Page London.

Sharp, H. e Hall, P. (2000) “An Interactive Multimedia Software House Simulation for Postgraduate Software Engineers”. Proceedings of the 22nd International Conference on Software Engineering. Limerick, Ireland, pp 688-691.

SEI - Software Engineering Institute. Performance Results of CMMI-Based Process Improvement. Pittsburgh: Software Engineering Institute, Carnegie Mellon University, 2006. Disponível em: <http://www.sei.cmu.edu/publications/documents /06.reports/06tr004.html>. Acesso em: 12 nov. 2007.

SOFTEX (2007) MPS.BR - Melhoria de Processo do Software Brasileiro - Guia Geral v1.2 Disponível em: < http://www.softex.br/mpsbr/_guias/MPS.BR_Guia_Geral_V1. 2.pdf>. Acesso em: 1 dez. 2008.

Wohlin, C., Runeson, P., Höst, M., Ohlsson, M. C., Regnell, B. e Wesslén, A. (2000) "Experimentation in Software Engineering - An Introduction”. Kluwer Academic Publishers, Norwell, MA. 